\title{
Kompetenznetzwerk Pädiatrische Onkologie und Hämatologie
}

In der Bundesrepublik Deutschland wird bisher das in Forschungseinrichtungen vorhandene Potential an wissenschaftlicher Innovation für die Lösung praktischer klinischer Probleme nicht ausreichend genutzt. Der Wissenstransfer aus der Grundlagenforschung in die Anwendungsforschung ist unzureichend, und forschungsrelevante Themen des medizinischen Alltags werden nur begrenzt in den Forschungseinrichtungen bearbeitet.

Aus diesen Gründen wurde vom Bundesministerium für Bildung und Forschung (BMBF) der Wettbewerb „Kompetenznetzwerke für die Medizin“ MedNet ausgeschrieben, um damit den Aufbau überregionaler Netzwerke für spezifische Krankheiten gezielt zu unterstützen. Insbesondere waren Krankheitsbilder gemeint, die durch eine hohe Morbidität oder Mortalität gekennzeichnet sind und als Modell für andere Krankheitsbereiche geeignet sind und/oder einen erheblichen Kostenfaktor darstellen.

Dieses Anliegen, eine Vernetzung zwischen Forschungsund Versorgungseinrichtungen effizient zu entwickeln, entspricht besonders den Bedürfnissen in der Pädiatrischen Onkologie und Hämatologie, für die eine Verbesserung der Kooperation und des Informationsaustausches zwischen Klinik und Forschung notwendig ist. Dadurch können die Qualität der klinischen Forschung in Verbindung mit der Grundlagenforschung angehoben und so die Diagnostik, die Therapie und letztlich die Heilungsraten und die Lebensqualität krebskranker Kinder verbessert werden.

Aus der Zusammenarbeit mehrerer Vorstands- und GPOH-Mitglieder entstand eine Projektskizze, die im Februar 1998 beim BMBF eingereicht wurde. Bei insgesamt 160 Netzwerkbewerbungen gelang den Autoren des GPOH-Projektes der Sprung in den engeren Kreis der 14 Teilnehmer, die aufgefordert wurden, im Sommer des letzten Jahres ein ausführliches Konzept auszuarbeiten.

\section{Das Konzept:}

Problematik: Die Behandlung von Krebserkrankungen im Kindesalter ist Hochleistungsmedizin, sie ist kostenintensiv, aber mit Heilungsraten von ca. $70 \%$ auch sehr effektiv. Die Behandlung erfolgt nach einheitlichen Therapieprotokollen, die auf den Ergebnissen von Vorläuferstudien basieren und damit die Prinzipien einer „Evidence-Based Medicine" erfüllen. Eine Verbesserung der Heilungsraten erfordert heute aufwendige und innovative Strategien. Der Erfolg wird nicht nur an Überlebensraten, sondern auch an der Vermeidung von Rezidiven, Zweittumoren und anderen Spätfolgen gemessen. Eine Optimierung der Therapie hat daher nicht nur wirtschaftliche, sondern auch gesellschaftpolitische Aspekte. Wegen der Seltenheit der Krebserkrankungen im Kindesalter und der aufwendigen Behandlung besteht die Notwendigkeit einer guten Kooperation und Kommunikation zwischen primär behan-

Klin. Pädiatr. 211 (1999) 187-188

(C) 1999 Georg Thieme Verlag Stuttgart - New York delnden Zentren, den Therapiestudiengruppen und den Arbeitsgruppen der Grundlagenforschung. Die essentiellen, personellen und technischen Voraussetzungen für den Informationsfluß und für die Rückkopplung zwischen den beteiligten Einrichtungen sind aber unzureichend. Darunter leidet die Generierung von Daten für Forschung und Therapieoptimierung, und die Umsetzung von Forschungsergebnissen wird verzögert.

Ziel unseres Antrages ist der Aufbau einer effizienten Forschungskooperation in folgenden Bereichen:

- Erforschung der genetischen Grundlagen für die Entstehung von Leukämien und bösartigen Tumoren und der Bedeutung von minimalen Resterkrankungen für die Prognose.

- Entwicklung neuer Therapieformen und Prüfung neuer Arzneimittel.

- Untersuchung von Akut- und Spätnebenwirkungen und der Lebensqualität, Verbesserung der Arzneimittelsicherheit.

- Verbesserung des Informationsflusses und der Kooperation zwischen Klinik und Forschung durch flächendeckende Verbreitung eines EDV-Systems.

- Beschleunigung des Informationsflusses zwischen den behandelnden Ârzten, den Studienzentralen und den Referenzzentralen durch telemedizinische Verfahren.

Netzwerkpartner sind neben dem GPOH-Vorstand das Deutsche Kinderkrebsregister, Kindertumorregister, Lymphknotenregister, Referenzzentren und Zentrale zur Erfassung von Spätfolgen, 21 Studienzentralen und alle Kliniken, in denen pädiatriatisch-onkologische Patienten behandelt werden, außerdem das Knochenmarktransplantationsregister und insbesondere das Institut für Medizinische Biometrie und Informatik der Universität Heidelberg, wo die Entwicklung des Dokumentationssystems für die Pädiatrische Onkologie (DOSPO) erfolgt.

Der Antrag wurde Ende Oktober beim Projektträger des BMBF eingereicht und im Januar 1999 von einer internationalen Jury bewertet. Parallel dazu wurden die 14 Netzwerke im Rahmen einer Ausstellung im Wissenschaftszentrum in Bonn präsentiert. Auf der Ausstellung haben wir unser Netzwerk mit den 12 Teilprojekten mit Dias, Postern und PC-Programm vorgestellt. Für diese Präsentation wurde in Zusammenarbeit mit Herrn Professor Pommerening, Mainz, eine Homepage entwickelt, die unter www.http://info.imsd.uni-mainz.de/GPOH auch nähere Einzelheiten zum Kompetenznetzwerk für die Pädiatrische Onkologie und Hämatologie enthält. Die Ausstellung wurde von der Jury besucht und war für Besucher und geladene Gäste geöffnet. Am Ende des zweiten Tages wurde von der Bundesministerin Frau Bulmahn bekanntgegeben, daß wir zu den 9 Gewinnern der Ausschreibung gehören. Das Kompetenznetzwerk für die Pädiatrische Onkologie und Hämatologie wird damit über einen Zeitraum von bis zu 5 Jahren mit ca. 4 Mio. DM pro Jahr gefördert. Im Antrag ist vorgesehen, daß die beteiligten Kliniken mit Dokumentar- oder Research-Nurse-Stellen unterstützt werden, um die für die Forschung notwendige Dokumentation und Materialgewinnung zu verbessern. 
Allen Kolleginnen und Kollegen, die ihre knappe Zeit für die aufwendigen Erstellung des Antrages geopfert haben, möchten wir an dieser Stelle ganz herzlich danken.

Prof. Dr. Ursula Creutzig Prof. Dr. H. Jürgens Prof. Dr. G. Henze

An der Vorbereitung des Antrages waren beteiligt:

\section{Ausarbeitung des Gesamtantrages:}

F. Berthold, U. Creutzig, W. Dörffel, G. Henze, H. Jürgens, P. Knaup-Gregori, C. Niemeyer, A. Reiter, M. Zimmermann

\section{Ausarbeitung der Teilprojekte:}

P. Knaup-Gregori, K. Pommerening, H. Jürgens, M. Paulussen, N. Graf, J. Boos, K.-M. Debatin, C. Niemeyer, J. Harbott, B. Dockhorn-Dworniczak, F. Berthold, S. Burdach, B. Zernikow, G. Calaminus, J. Beck, J. Michaelis 